\title{
Mit vielen einzigartigen Vorteilen
}

Das aktuelle Programm der neuen Premium-Prophylaxe-Marke Lunos $®$ reicht von der Mundspüllösung, dem Pulverstrahlhandstück mit Wechselkammerprinzip über unterschiedliche Pulver und Pasten bis hin zum Wellness-Tuch. Das Sortiment besteht aus einer Vielzahl an Produkten, die zusammen ein schlüssiges System bilden Ein Beispiel stellt das Pulverstrahlhandstück MyFlow dar. Durch das Wechselkammerprinzip bietet es dem Team einen besonderen Komfort: Der Pulverbehälter lässt sich einfach austauschen, womit das lästige Nachfüllen während der Anwendung am Patienten entfällt. Das Handstück arbeitet mit verschiedenen Prophylaxepulvern. Die Variante Gentle Clean von Lunos enthält neuartige Abra-

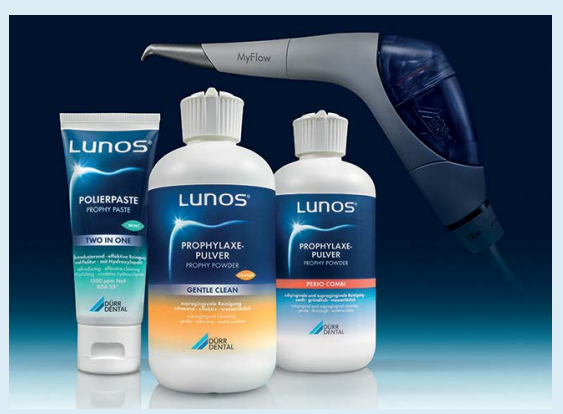

sivkörper auf der Basis des nicht kariogenen Disaccharids Trehalose für die sanfte Reinigung im supragingivalen Bereich und ist in 3 Geschmacksrichtungen verfügbar (Spearmint, Orange, Neutral). Alternativ dazu kann Lunos Prophylaxepulver Perio Combi für supra- und subgingivale Maßnahmen eingesetzt werden. Die Lunos Prophylaxe-Linie umfasst außerdem folgende Produkte: Fluoridlack, Fluorid Gel, die hochgefüllte Fissurenversiegelung auf Kompositbasis, das ProphyKissen aus einem Tempur-ähnlichen Schaum zur bequemen Lagerung des Patienten und das hautpflegende ProphyWellnesstuch mit Aloe Vera.

Nach einer Pressemitteilung der Dürr Dental AG, Bietigheim-Bissingen www.duerrdental.com 